\title{
Géométrie interne du "Salvator Mundi" (version dite Cook, attribuée à Léonard de Vinci)
}

\section{Internal geometry of "Salvator Mundi" (so-called Cook version, attributed to Leonardo da Vinci)}

\author{
Jean-Pierre Crettez ${ }^{1}$ \\ ${ }^{1}$ Chercheur émérite à Télécom-Paristech, jean-pierre.crettez@wanadoo.fr
}

\begin{abstract}
RÉSUMÉ. L'étude présentée ici concerne la recherche de la géométrie interne du Salvator Mundi (version dite Cook, attribuée à Léonard de Vinci), suivie, par comparaison, de celle de la version dite de Ganay. Cette approche confirme la méthodologie que nous avons développée précédemment ${ }^{1,2}$. Elle met en évidence la démarche créatrice de Léonard et en particulier sa conception dynamique de la géométrie.

ABSTRACT. The study presented here concerns the search for the internal geometry of the Salvator Mundi (so-called Cook version, attributed to Leonardo da Vinci), followed, by comparison, with that of the so-called Ganay version. This approach confirms the methodology we developed previously ${ }^{2,3}$. It highlights Leonardo's creative approach and in particular his dynamic conception of geometry.

MOTS-CLÉS. architecture, commensurabilité, construction interne, géométrie interne, géométrie secrète, maillage harmonique, entrelacs, poncif, filigrane d'or, formes consonantes visuelles, stylisation des formes.

KEYWORDS. architecture, commensurability, internal construction, internal geometry, secret geometry, harmonic mesh, interlacing, pounced drawing, gold filigree, visual consonant forms, stylization of forms.
\end{abstract}

\section{Introduction}

Les peintres établissent leur composition à l'aide de la géométrie interne qui leur permet de dresser les lignes directrices, d'établir les relations entre les éléments picturaux, et d'idéaliser en partie leur forme à l'aide d'un compas ou d'un ellipsographe. Cependant, la géométrie interne n'est pas tracée directement sur la fresque ou sur la toile, mais de préférence sur un patron. Sur ce patron, les peintres commencent par tisser une trame géométrique régulière que nous avons appelée maillage. Le maillage carré ou le plus souvent le maillage harmonique sert de support au tracé de la géométrie interne. Dans un maillage harmonique chaque maille est un petit rectangle harmonique dont les côtés sont respectivement proportionnels à $\mathbf{1}$ et $\sqrt{ } \mathbf{2}$, et sa diagonale à $\sqrt{ } \mathbf{3}$.

Lorsque l'étude de la composition est achevée, il est alors inutile de transférer le maillage et les lignes de construction, seules les contours des formes sont transférées sur la toile ou sur le panneaau de bois par la méthode du poncif. Les contours des formes tracées sur le patron, sont percés de petits trous. Le passage d'une ponce à travers ces trous reproduit simplement les contours en pointillé sur la toile ou sur un autre support. Par extension, certains appellent poncifs ces patrons percés de petits trous. Les poncifs permettent au maître ou à ses disciples d'effectuer des répliques. C'est pourquoi, le tracé du maillage et celui de la géométrie interne n'apparaissent pas dans l'œuvre peinte, ni dans son image radiographique, et ni dans son image infra-rouge : la géométrie interne demeure secrète. Mais, lorsque celle-ci a été tracée en se référant aux nœuds du maillage harmonique, les éléments picturaux issus de la géométrie interne sont quantifiés, la géométrie interne devient discrète. Détecter la géométrie interne, c'est retrouver dans le non-visible, les éléments de construction du visible ${ }^{3}$.

\footnotetext{
1 J-P. Crettez: [2] Les supports de la géométrie interne des peintres: de Cimabue à G. de La Tour. Editions ISTE (2017).

2 J-P. Crettez: [3] Openscience -Géométrie interne d'une "Nuit" de G. de La Tour: "L'Apparition de l'ange à saint Joseph" (musée des beaux-arts, Nantes) vol 2 -Numero 1

3 Ibid.

(C) 2019 ISTE OpenScience - Published by ISTE Ltd. London, UK - openscience.fr 
Inversement, lorsqu'une œuvre a été créée par un peintre sur un maillage carré ou harmonique, le caractère discret de la géométrie interne rend possible et crédible la détection des points particuliers, des éléments linéaires, et des courbes particulières comme les formes elliptiques. 11 permet de déceler le maillage avec précision. Grâce à ce dernier, il devient possible de détecter les autres éléments picturaux, de retrouver la géométrie interne de la composition et ainsi de mettre en évidence la démarche créatrice du peintre

En appliquant cette méthodologie, nous avons pu mettre en évidence dans notre livre, le maillage et la géométrie interne de cinq portraits ${ }^{4}$ de femme, peints par Léonard de Vinci, dont celui de la Joconde.

\section{Particularités des œuvres de L. de Vinci.}

L'analyse de ces cinq portraits met bien en évidence la démarche suivie par Léonard pour construire ses portraits. Même si son style a évolué au cours de sa vie artistique, sa démarche est restée la même. Connaissant ses qualités de dessinateur comme le montre l'étude réalisée pour le Salvator Mundi (figure 2), nous aurions pu penser que le tracé du contour supérieur de la tête de chacun de ces cinq personnages, était le résultat d'un élan instinctif, mais lorsque chacun de ces contours correspond de façon précise à un arc d'ellipse (de longueur assez importante, presque une demi-ellipse) centré sur l'axe vertical médian, au point correspondant au centre de gravité de la tête, et tracé avec précision sur un maillage, nous devons admettre que l'approche empruntée par Léonard de Vinci pour composer ses cinq portraits est une approche géométrique.

Pour Léonard, la géométrie naît du mouvement. "Il est significatif que contre la conception statique de la géométrie albertienne - pour laquelle les points juxtaposés forment la ligne tandis que les lignes réunies (comme les fils d'une toile) forment la surface - Léonard développe la théorie dynamique de la géométrie: "c'est le mouvement du point qui engendre la ligne, le mouvement transversal de la ligne qui engendre la surface, et le corps est fait par le mouvement ${ }^{5}{ }^{6} "$.

En effet, la géométrie permet à Léonard de modéliser, de façon parfaite, par des courbes géométriques simples, les formes qu'il observe dans la nature, comme la forme hélicoïdale des coquilles de nautiles, la répartition des branches et des feuilles des arbres... Car, pour Léonard, ces formes créées par la nature ne sont pas aléatoires, elles sont dues à la Nécessité. "Nécessité est maîtresse et tutrice de la Nature. Nécessité est le thème et l'artificier de la nature - le frein, la loi et le thème $e^{7 "}$. Elle agit de façon performante en adaptant parfaitement la forme à la fonction. " $\hat{O}$ miraculeuse, ô stupéfiante Nécessité, toi dont la loi contraint tous les effets à naître de leurs causes, par la voie la plus brève 8 !"

Pour effectuer ses portraits, Léonard élabore sa composition sur un patron qu'il subdivise à l'aide d'un maillage: un maillage carré pour le portrait de Ginevra de' Benci, un maillage harmonique pour les quatre autres. Sur ce maillage, il trace l'une des ellipses particulières ${ }^{9}$ qui s'adapte le mieux au profil recherché du personnage, en la centrant sur l'axe médian et en l'orientant suivant la posture que doit prendre le modèle. Ensuite, Léonard, poursuivant la dynamique du tracé, assure la continuité du contour en s'écartant insensiblement du tracé elliptique afin de dessiner avec minutie le pourtour du visage. "Léonard de Vinci, en recherchant la dynamique de la formation du visage, idéalise le portrait, en imposant aux variations de la nature, la pureté et la beauté d'une forme pure, qu'il

\footnotetext{
4 [2]. §. 8.2.3.

5 Codex Arundel, $159 \mathrm{r}$

${ }^{6}$ D. Arasse : [1] Leonard de Vinci p. 16

${ }^{7}$ Codex Forster III, $43 v$

${ }^{8}$ Codex Atlanticus. 345 v. $b$

9 [2]. §. 2.4.4.
}

(c) 2019 ISTE OpenScience - Published by ISTE Ltd. London, UK - openscience.fr 
s'empresse paradoxalement de dissimuler à l'aide de sa technique qu'il a mise au point: le sfumato qui conduit à l'invisibilité effective du contour: Les limites latérales de ces corps sont constituées par la ligne de surface, ligne d'une épaisseur invisible. ${ }^{10}{ }$

Dans ces cinq portraits, Léonard cherche à montrer le tempérament du modèle, et en particulier sa vie intérieure. Dans une note du codex Atlanticus, il précise : "Une peinture ou toute représentation de figure doit être de façon que ceux qui la voient puissent aisément connaître, par les attitudes, les mouvements de l'âme ${ }^{11}$." Pour donner vie au personnage, Léonard donne à ses élèves le conseil suivant :" Tu ne feras jamais les têtes droites sur les épaules, mais tournées de côté, à droite ou à gauche, même si elles regardent en haut ou en bas, ou tout droit, parce qu'il est nécessaire de faire en sorte que leurs mouvements aient l'air d'être vivants et non figés ${ }^{12} . "$

Le Salvator Mundi nous regarde de face: ce n'est pas un portrait. C'est une représentation : la représentation du Christ. Néanmoins, Léonard a étudié sa composition, en suivant la même démarche géométrique que celle exposée ci-dessus.

\section{Présentation du Salvator Mundi version Cook}

Le tableau (figure 1) représente le Christ rédempteur ${ }^{13}$ dans la version dite Cook. Peint vers 1506, il est contemporain de la Joconde, portrait réalisé par Léonard à partir de 1503 sur plus d'une dizaine d'années. C'est une peinture à l'huile sur un panneau constitué d'une seule planche de noyer qui a pour dimensions : $65,6 \mathrm{~cm}$ de hauteur et $45,4 \mathrm{~cm}$ de largeur.

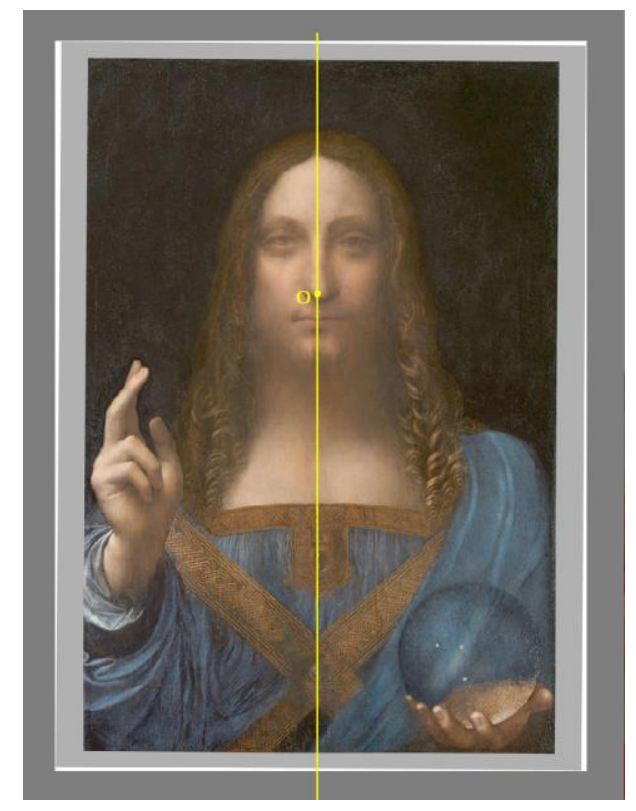

Figure 1. L de Vinci : Salvator Mundi ${ }^{14}$ (version dite Cook) (musée du Louvre, Abou Dabi). Le tableau a pour dimensions : $65,6 \mathrm{~cm}$ de hauteur et $45,4 \mathrm{~cm}$ de largeur.

L'œuvre a été apportée en Angleterre par Henriette Marie de France lors de son mariage avec Charles $1^{\mathrm{er}}$. En 1763, le tableau est vendu aux enchères par le fils du duc de Buckingham. Il réapparaît en 1900, quand il est acheté par un collectionneur britannique, Francis Cook, vicomte de Monserrate.

\footnotetext{
10 [2]. §. 8.2.3.

${ }^{11}$ C. A. 139 r.d, Codex Atlanticus, conservé à la Bibliothèque Ambroisienne de Milan.

12 Léonard de Vinci, Le traité de peinture, note 354, p. 149. Editions Jean de Bonnot, Paris 1982.

13 Le Salvator Mundi est un thème de l'art chrétien, inspiré du Christ pantocrator, popularisé par l'art byzantin, par Simone Martini (1315), puis par les peintres d' Europe du Nord, comme Jan van Eyck, Hans Memling.

14 https://commons.wikimedia.org/wiki/File:Leonardo_da_Vinci,_Salvator_Mundi,_c.1500,_oil_on_walnut,_45.4_\%C3\%97_65.6_cm.jpg

(c) 2019 ISTE OpenScience - Published by ISTE Ltd. London, UK - openscience.fr 
Réapparu et restauré en 2005, il est rendu public en 2011 lors d'une exposition à la National Gallery (Londres) et authentifié par Martin Kemp comme une œuvre ${ }^{15}$ de L. de Vinci.

Le Christ est représenté frontalement sur un fond sombre. «Symmetry and frontality were more or less obligatory when Christ is depicted as savior of the world. ${ }^{16} \gg \mathrm{Il}$ porte une tunique bleu-mauve finement brodée avec des garnitures en brocart d'or, formant sur sa poitrine comme une étole avec deux bandes croisées, ornées de motifs géométriques répétitifs. Il a de longs cheveux bouclés. Ses yeux tournés vers le devant, offrent au fidèle une confrontation directe avec le divin.

De sa main droite levée, il donne la bénédiction avec l'index et le majeur croisés, évoquant la double nature (humaine et divine) du Christ ${ }^{17}$. Sa main gauche ne s'appuie pas sur un livre. Elle porte un globe, mais il ne s'agit pas d'un globe terrestre surmonté d'une croix comme on le voit souvent, mais d'une sphère de cristal transparente; c'est l'univers tout entier (quelques étoiles y sont incrustées) que le Christ tient dans sa main. «So what you've got in the "Salvator Mundi" is really "a savior of the cosmos", and this is a very Leonardesque transformation ${ }^{18} »$.

Cependant, le Christ semble à l'étroit dans ce tableau. L'ouverture de ses bras ne correspond pas à la largeur de ses épaules. Sa main gauche, malgré l'importance symbolique de la sphère de cristal qu'elle supporte, est confinée dans le coin inférieur droit du tableau. Et sur le côté gauche, l'avant-bras droit qui a pourtant fait l'objet d'une étude préalable (figure 2) n'est pas entièrement représenté. Il faut donc admettre que le format de la composition étudiée par le maître, devait être plus grand et plus large que celui du tableau actuel.

\section{Recherche du maillage de la composition}

Pour déterminer la géométrie interne du tableau, il n'est donc pas possible de se fier à son cadre. On ne peut que s'appuyer sur des éléments caractéristiques présents dans l'œuvre elle-même. Deux éléments picturaux nous semblent essentiels (figure 2) : les bandes croisées sur la poitrine, la tête du Christ. Le Salvator parait légèrement incliné vers la droite. Une légère rotation $\left(\sim 0.5^{\circ}\right)$ permet de rétablir pleinement la frontalité, de telle sorte que l'axe vertical médian passe exactement par le milieu du visage et par le centre du croisement des deux bandes.

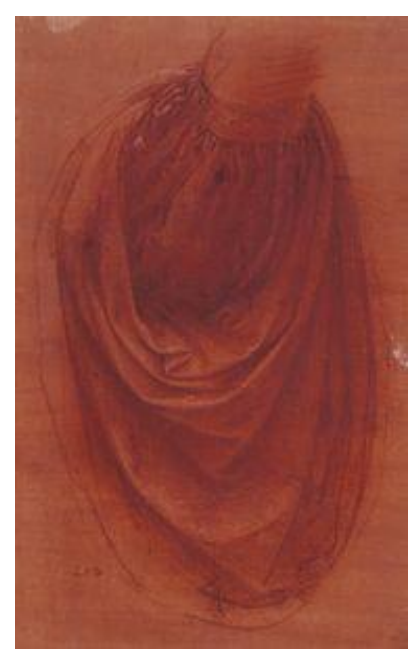

Figure 2. Étude pour le Salvator Mundi (royal collection famille britannique).

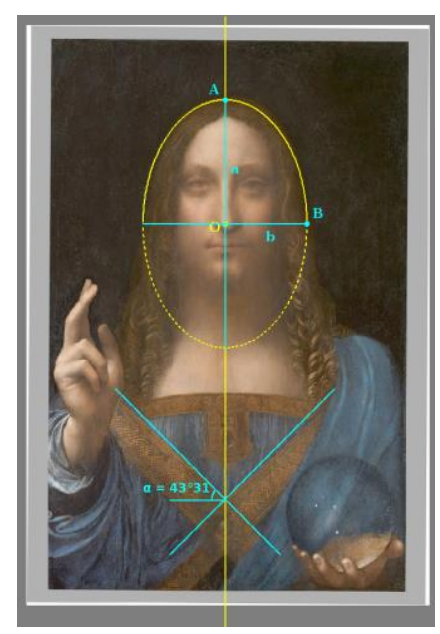

Figure 3. Le contour du crâne a la forme d'un arc d'ellipse.

\footnotetext{
15 En 2017 le tableau est acquis par le prince d'Arabie saoudite lors d'une vente aux enchères à New-York. Il devait être exposé au Musée du Louvre d'Abou Dabi.

${ }^{16}$ Kemp M.:[4], Leonardo, p. 208.

${ }^{17}$ Sabine Gignoux, «Le Salvator Mundi de Léonard de Vinci décrypté par François Bœspflug», sur la-croix.com, 14 novembre 2017

18 Andrew M. Goldstein, The Male «Mona Lisa»? : Art Historian Martin Kemp on Leonardo da Vinci's Mysterious "Salvator Mundi», Blouin Artinfo, 17 novembre 2011. 
Plus précisément, les deux bandes de l'étole (figure 3) ne se croisent pas à $\mathbf{4 5}^{\circ}$, mais elles sont plus précisément inclinées symétriquement par rapport à l'axe horizontal suivant un angle $\alpha=43^{\circ} 31$, dont la tangente est égale à $\mathbf{2} \sqrt{\mathbf{2}} / \mathbf{3}$.

Suivant la méthode exposée plus haut, Léonard a donné au contour du crâne (figure 3) la forme d'un arc d'ellipse supérieur à $160^{\circ}$. Respectant la symétrie frontale, le grand axe de l'ellipse est placé verticalement, et il est confondu avec l'axe vertical médian du tableau. Le centre $\mathbf{O}$ de cette ellipse, est situé précisément à l'extrémité du nez. Cette ellipse ${ }^{19}$ a pour demi-grand axe $\mathbf{a}=\mathbf{O A}$, pour demi-petit axe $\mathbf{b}=\mathbf{O B}$ et pour distance focale $\mathbf{f}$ telle que $\mathbf{f}^{2}=\mathbf{a}^{\mathbf{2}}-\mathbf{b}^{2}$. Les côtés $\mathbf{O A}$ et $\mathbf{O B}$ sont tels que $\mathbf{O A}$ $=\mathbf{O B} \sqrt{2}$, comme on peut le vérifier (figure 4) à l'aide de la diagonale du carré. Par suite, le rectangle AОBC est un rectangle harmonique. Puisque $\mathbf{a}=\mathbf{b} \sqrt{\mathbf{2}}$, on en déduit que $\mathbf{f}=\mathbf{b}$. C'est une ellipse particulière.

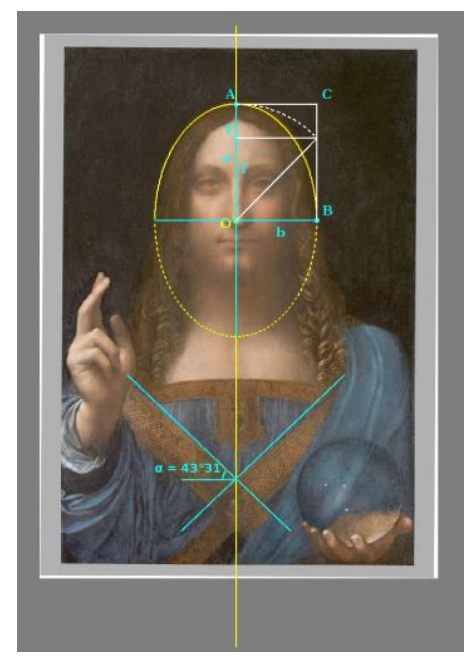

Figure 4. Les demis-axes ( $a$ et b) de cette ellipse forment un rectangle harmonique.

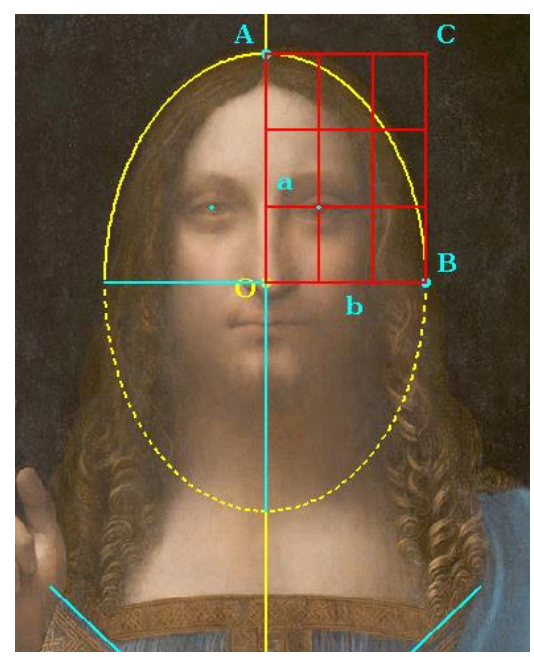

Figure 5. La subdivision (3×3) de ce rectangle détermine un maillage harmonique vertical.

La subdivision ( $3 \times 3)$ du rectangle harmonique AOBC détermine (figure 5) un maillage harmonique vertical. L'intersection de la première ligne et la première colonne de ce maillage coïncide précisément avec le centre de la pupille de son œil gauche.

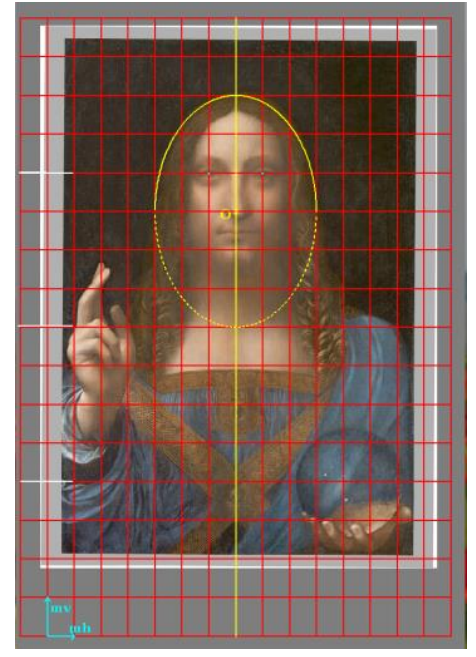

Figure 6. Maillage harmonique vertical (16x16) servant de support à la composition.

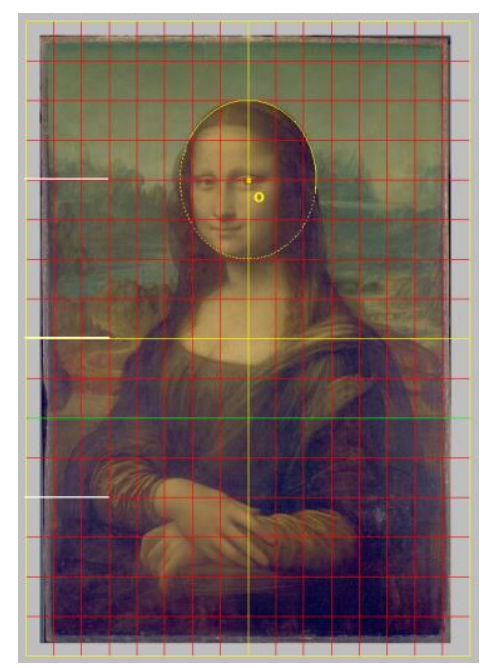

Figure 7. Maillage harmonique vertical (16x16) servant de support à la composition. 
Cette cohérence nous incite à étendre ce maillage à l'ensemble du tableau (figure 6). Elle nous permet ainsi de déterminer rapidement le maillage qui sert de support à la composition. Le maillage est un maillage harmonique vertical (16x16) (figure 6) qui recouvre tout le tableau, mais dépasse physiquement ses bords, notamment vers le bas. La maille a pour hauteur $\boldsymbol{m}_{v}$ et pour largeur $\boldsymbol{m}_{\boldsymbol{h}}$, avec la relation $\boldsymbol{m}_{v}=\boldsymbol{m}_{\boldsymbol{h}} \sqrt{\mathbf{2}}$.

\section{Format de la composition}

En se référant aux dimensions physiques du tableau nous pouvons estimer, grâce à la quantification, que la composition étudiée par Léonard devait avoir une largeur de l'ordre de $56,7 \mathrm{~cm}$ et pour hauteur de l'ordre de $80,2 \mathrm{~cm}$. La maille aurait pour hauteur $\boldsymbol{m}_{v}=5,0 \mathrm{~cm}$ et pour largeur $\boldsymbol{m}_{\boldsymbol{h}}=3,5 \mathrm{~cm}$. Il faut remarquer que le format de la composition est très proche du format de la composition ${ }^{20}$ (figure 7) que nous avions mis en évidence dans l'étude de la Joconde, et pour laquelle nous avions estimé une largeur de 57,5cm ( 1 braccio) et une hauteur de 81,3cm. Dans ces deux compositions, la hauteur des yeux du personnage est située aux trois quarts de la hauteur de la composition (figure 7).

\section{Éléments de la géométrie interne du Salvator Mundi version Cook}

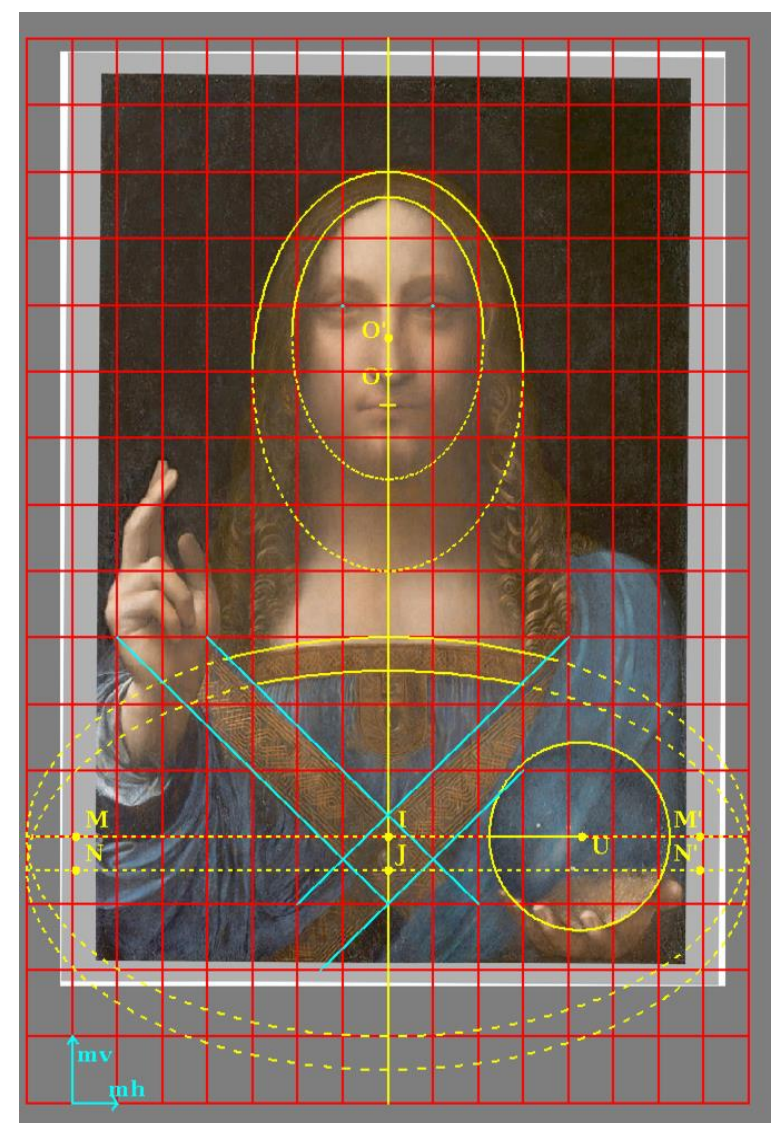

Figure 8. Géométrie interne du Salvator Mundi version Cook

Par suite de la symétrie frontale, chaque pupille est centrée (figure 8) sur un nœud du maillage. La distance entre les deux pupilles est égale à $2 \boldsymbol{m}_{h}$. Cette distance est $\sqrt{ } \mathbf{2}$ plus grande que la distance $\boldsymbol{m}_{v}$ qui existe entre les deux pupilles de la Joconde (figure 10). Mais la tête de la Joconde est tournée de trois quarts vers la droite de telle sorte que l'axe vertical médian passe par son œil gauche.

Le contour du visage du Salvator présente la forme d'un arc d'ellipse semblable à celui du crâne. Il est centré sur l'axe vertical médian au point O', une demi-hauteur de maille au-dessus du premier. 
L'ouverture de la bouche est située une demi-hauteur de maille au-dessous du nez.

En haut de la tunique, la collerette en filigrane d'or est limitée par deux arcs d'ellipse égaux, décalés d'une demi-hauteur de maille. La première est centrée en $\mathbf{I}$, au quart de la hauteur de la composition. Les deux ellipses sont tracées sur le maillage. Elles ont pour paramètres: $\mathbf{a}=\mathbf{8} \boldsymbol{m}_{\boldsymbol{h}}, \mathbf{b}=\mathbf{3} \boldsymbol{m}_{\boldsymbol{v}}, \mathbf{f} \cong \mathbf{7} \boldsymbol{m}_{\boldsymbol{h}}$.

Les bords des deux bandes croisées sur la poitrine sont limitées par des droites tracées entre les nœuds du maillage. En se croisant les deux bandes forment un pseudo-carré dont la diagonale vaut $\mathbf{2 m}_{\boldsymbol{h}}$ . La largeur des bandes est de l'ordre de la hauteur de la maille: $\mathbf{2}_{\boldsymbol{h}} / \sqrt{\mathbf{2}}=\boldsymbol{m}_{\boldsymbol{v}}$. L'ornementation de ces bandes sera analysée par la suite.

La sphère de cristal est centrée au point $\mathbf{U}$, au quart de la hauteur de la composition. Elle a pour rayon $\mathbf{r}=\mathbf{2} \boldsymbol{m}_{\boldsymbol{h}}$. Curieusement, elle n'est pas centrée sur un nœud du maillage suivant la logique de Léonard, mais à une distance telle que $\mathbf{I U}=\mathbf{4 , 2 4} \boldsymbol{m}_{h}$. Par raison de symétrie avec les doigts de la main droite, elle devait se trouver plus à droite à 5 mailles de l'axe vertical médian. Lors du transfert du patron, le peintre aurait déplacé horizontalement cette partie du poncif vers cet axe, pour que la main gauche et la sphère de cristal soient toutes les deux, contenues dans la planche de noyer.

Ainsi, ayant disposé tous ces éléments géométriques sur le patron, le peintre poursuivait la dynamique du tracé, achevait le pourtour du visage, dessinait les autres éléments picturaux, puis perçait les contours de petits trous pour constituer le poncif.

\section{Précision sur les ellipses de la tête du Salvator Mundi}

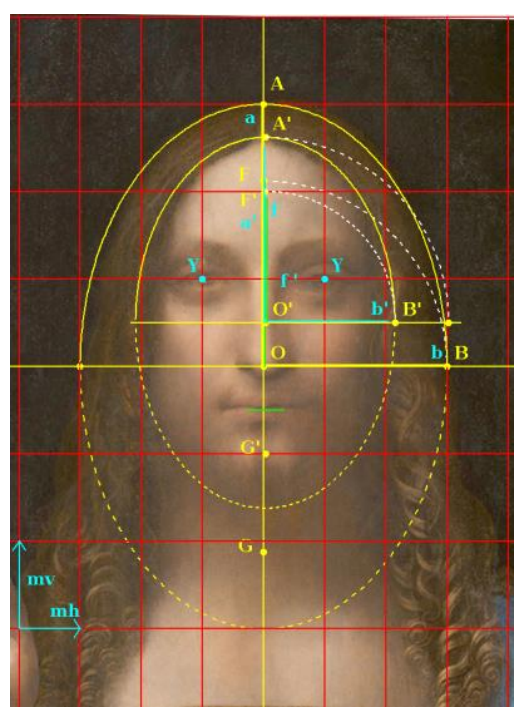

Figure 9. Géométrie interne de la tête du Christ.

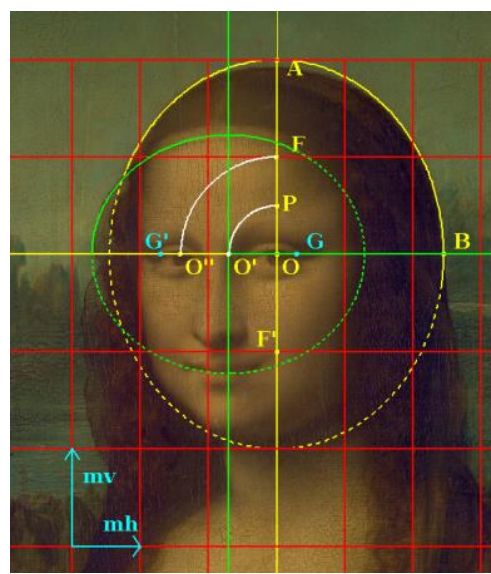

Figure 10. Géométrie interne de la tête de la Joconde.

Comme nous l'avons vu précédemment, l'ellipse qui a permis de modéliser le haut du crâne est centrée en $\mathbf{O}$, elle est orientée verticalement. Ses paramètres (figure 9) sont définis sur le maillage: le demi-grand axe $\mathbf{O A}$ vaut $\mathbf{a}=\mathbf{3} \boldsymbol{m}_{v}$, le demi-petit axe $\mathbf{O B}$ vaut $\mathbf{b}=\mathbf{3} \boldsymbol{m}_{h}$, et la distance focale $\mathbf{f}=\sqrt{ }\left(\mathbf{a}^{2}\right.$ $\left.\mathbf{b}^{2}\right)=\mathbf{3} \boldsymbol{m}_{h}$ est égale au demi-petit axe: $\mathbf{f}=\mathbf{b}$. Son excentricité vaut $\boldsymbol{\varepsilon}=\mathbf{f} / \mathbf{a}=\boldsymbol{m}_{h} / \boldsymbol{m}_{v}=\mathbf{1} / \sqrt{\mathbf{2}}=\mathbf{0 , 7 0 7}$.

L'ellipse qui initialise le contour du visage, est centrée en $\mathbf{O}$ ', elle est orientée verticalement. Ses paramètres (figure 9) sont aussi définis sur le maillage : le demi-grand axe $\mathbf{O A}^{\prime}$ vaut $\mathbf{a}^{\prime}=\mathbf{3}_{\boldsymbol{h}}$, le demi-petit axe $\mathbf{O B}$ vaut $\mathbf{b}^{\prime}=\mathbf{3} \boldsymbol{m}_{v} / \mathbf{2}$, et la distance focale $\mathbf{f}^{\prime}=\sqrt{ }\left(\mathbf{a}^{, 2}-\mathbf{b}^{\mathbf{2}}\right)=\mathbf{3} \boldsymbol{m}_{v} / \mathbf{2}$ est aussi égale au demi-petit axe: $\mathbf{f}^{\prime}=\mathbf{b}^{\prime}$. Son excentricité vaut $\boldsymbol{\varepsilon}^{\prime}=\mathbf{f}^{\prime} / \mathbf{a}^{\prime}=\boldsymbol{m}_{h} / \boldsymbol{m}_{\boldsymbol{v}}=\mathbf{1} / \sqrt{\mathbf{2}}=\mathbf{0 , 7 0 7}$. Ces deux ellipses sont 
du même type. Ce sont, suivant notre classification ${ }^{21}$, des ellipses de type 1 . Nous avions appelé ellipse "pure", cette ellipse de type 1.

\section{Consonance visuelle}

Les peintres, sensibles à l'analogie entre les harmonies visuelles et les harmonies sonores ont cherché à modéliser certains éléments picturaux par des formes visuelles consonantes. Par définition, deux formes visuelles sont consonantes lorsqu'elles sont semblables et lorsque le rapport de leur surface est égal à l'un des rapports musicaux $(1,1 / 2,2 / 3,3 / 4)$ : l'unisson, l'octave, la quinte, la quarte.

Les ellipses de la tête et du visage du Salvator sont semblables. Leurs excentricités vérifient: $\boldsymbol{\varepsilon}^{2}+\boldsymbol{\varepsilon}^{\prime 2}$ $=\mathbf{1}$ : elles sont complémentaires ${ }^{22}$. La seconde est $\sqrt{\mathbf{2}}$ fois plus petite que la première: $\mathbf{a} / \mathbf{a}=\mathbf{b}^{\prime} / \mathbf{b}=$ $\mathbf{1} / \sqrt{ } \mathbf{2}$. Sa surface est donc deux fois plus petite. Elles sont consonantes, et résonnent à l'octave.

Cette consonance visuelle recherchée par Léonard, rappelle celle qu'il avait déjà introduite dans la construction de la Joconde. En effet, dans l'étude de la géométrie interne de la Joconde, nous avions mis en évidence deux formes elliptiques, tracées sur un maillage harmonique: l'une pour modéliser le contour de la tête, l'autre pour délimiter (figure 10) le bord du voile de gaze transparent qui recouvre sa tête, et qui passe par la commissure des lèvres. Elles sont plus arrondies que celles du Salvator. Elles ont une même excentricité: $\boldsymbol{\varepsilon}=\boldsymbol{\varepsilon}^{\prime}=\mathbf{0 , 5}$. La seconde est $\sqrt{ } \mathbf{2}$ fois plus petite que la première. Sa surface est donc deux fois plus petite. Elles ne sont pas complémentaires, mais elles sont consonantes entre elles, et résonnent à l'octave. Toutefois, leurs grands axes ne sont pas parallèles mais sont orthogonaux.

\section{Propriétés de l'ellipse de type 1}

Pour modéliser la tête du Salvator mundi, à la fois homme et dieu, Léonard ne pouvait choisir qu'une ellipse particulière, idéale, unique. Comme nous l'avons remarqué, il a choisi pour la tête et le visage, un même type d'ellipse: une ellipse de type 1, dont la distance focale est égale au demi-axe : $\mathbf{f}=$ $\mathbf{b}$, et d'excentricité $\mathbf{1} / \sqrt{ } \mathbf{2}=\mathbf{0 , 7 0 7}$. Cette ellipse se trouve dans l'ensemble des ellipses, précisément à la limite entre les ellipses allongées $(\mathbf{f}>\mathbf{b})$ et les ellipses arrondies $(\mathbf{f}<\mathbf{b})$. Elle est égale à sa complémentaire. Elle possède plusieurs propriétés géométriques.

Cette ellipse de type 1 qui est telle que $\mathbf{a}=\mathbf{b} \sqrt{ } \mathbf{2}$, peut être inscrite dans un rectangle harmonique: elle est ainsi facile à représenter sur un maillage harmonique. Dans cette ellipse, on peut inscrire un cercle passant par les foyers, ou encore un carré sur la pointe (figure 11a).

Le petit axe b de l'ellipse de la tête est égal au grand axe a' de l'ellipse du visage, ainsi l'ellipse du visage peut être entièrement inscrite dans l'ellipse de la tête (figure 11b). La grande ellipse est alors divisée en trois parties : une ellipse interne située entre deux croissants de lune. La surface de cette ellipse interne est égale à la somme de la surface des deux croissants de lune.

Enfin, dans cette petite ellipse de type 1 , on peut aussi inscrire une ellipse de type $1, \sqrt{ } \mathbf{2}$ fois plus petite, et par suite constituer une suite d'ellipses de type 1, consonantes à l'octave (figure 11c). 

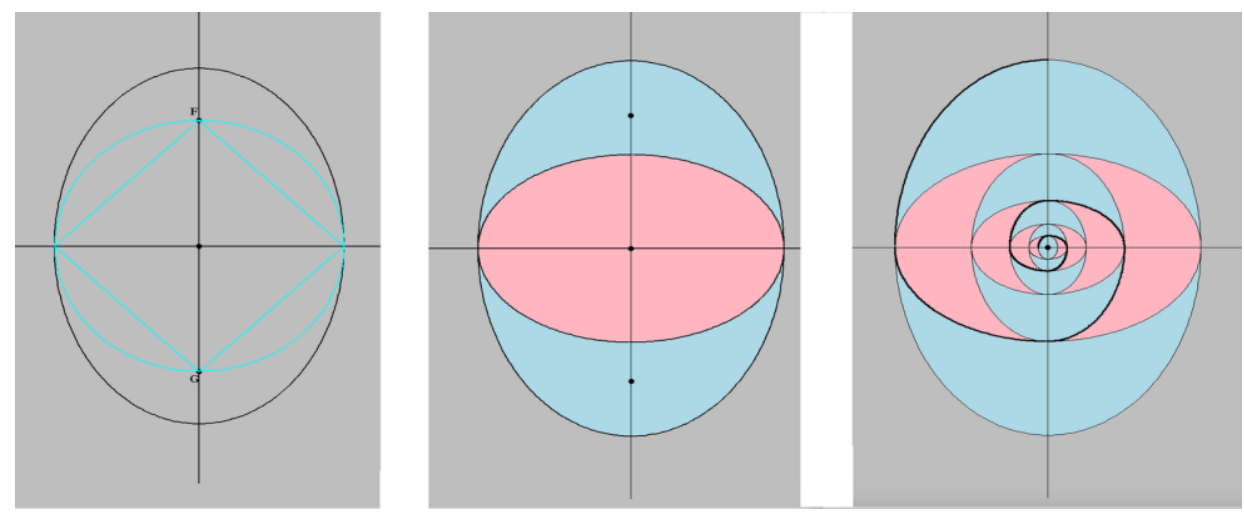

Figure 11. Propriétés de l'ellipse de type 1 modélisant la tête du Salvator. Fig. 11a : elle peut contenir un cercle passant par les foyers et un carré sur la pointe. Fig. 11b: dans cette ellipse, on peut inscrire une ellipse $\sqrt{ } 2$ fois plus petite. Fig. $11 \mathrm{c}:$ suite d'ellipses de type 1 consonantes à l'octave.

\section{Autres applications de cette ellipse de type 1}

Léonard connaissait bien cette ellipse de type 1, puisque seize ans plus tôt, lors de son séjour à Milan, entre 1488 et 1490, il avait déjà déterminé le contour de la tête de la Dame à l'hermine l' $^{23}$ l'aide de cette même ellipse, orientée suivant la diagonale de la maille.

Et dix ans plus tôt, avec cette même ellipse, orientée suivant la diagonale de deux mailles adjacentes, il avait donné forme au contour de la tête de la Belle Princesse ${ }^{24}$

Cent trente ans plus tard, Georges de La Tour cherchant la stylisation des formes, eut lui aussi recours aux formes idéalisées par des ellipses, en particulier par cette même ellipse de type 1.

L'image sous-jacente de la tête de l'Enfant dans la radiographie du Saint Joseph charpentier, présente un contour qui peut être approché par cette ellipse ${ }^{25}$.

Dans l'Apparition de L'Ange à Saint Joseph ${ }^{26}$, le contour de la tête de l'Ange et et celui de la tête de Joseph sont modélisés par ce même type d'ellipse. Ces deux ellipses tracées sur un maillage harmonique, sont égales et orientées symétriquement suivant la diagonale de la maille. Elles sont complémentaires, et elles sont consonantes à l'unisson.

G. de La Tour nous a laissé une œuvre tardive, peinte vers 1651: Saint-Jean Baptiste dans le désert $^{27}$. La forme elliptique de la chevelure est évidente, ainsi que celle de son dos courbé. Les deux ellipses sont verticales, elles ont même excentricité $\boldsymbol{\varepsilon}=\boldsymbol{\varepsilon}^{\prime}=\mathbf{0 , 7 0 7}$. Elles sont de type 1. Leurs dimensions sont dans le rapport 2/5, et leurs surfaces dans le rapport 4/25. Elles sont commensurables.

\section{L'ornementation des deux bandes croisées de l'étole}

Les deux bandes de l'étole ne se superposent pas: ce n'est pas une vraie étole. Elles ne se croisent pas. Elles s'entrecroisent. Elles sont tissées dans la tunique que porte le Salvator Mundi. Leur surface est recouverte d'une ornementation prodigieusement complexe, constituée d'une longue cordelette de fil d'or (un filigrane) qui se repliant sur elle-même, forme des entrelacs. Léonard était passionné par les entrelacs, probablement depuis son séjour dans l'atelier de Verrocchio.

[2]. §. 8.2.3.2

[2]. \$. 8.2.3.3

[2]. \$. 9.3.

[3].

27 [2]. §. 9.6. 


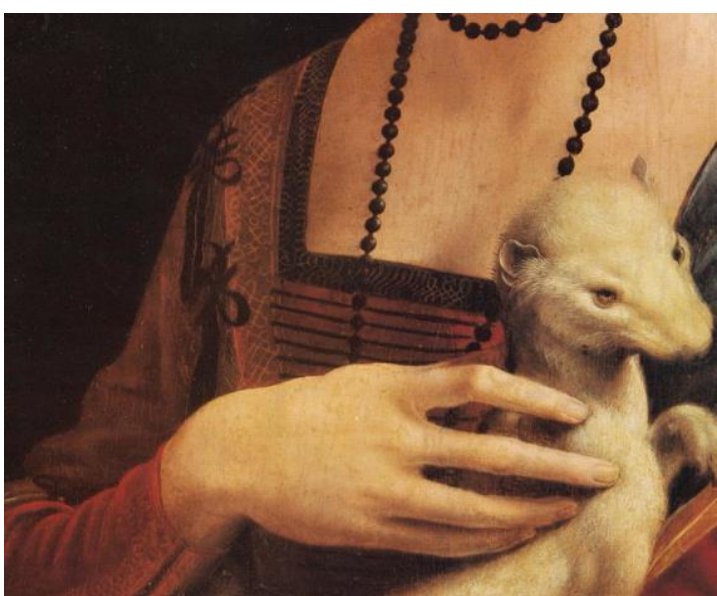

Figure 12. La Dame à l'hermine : (détail) une bande d'entrelacs descend de l'épaule droite.

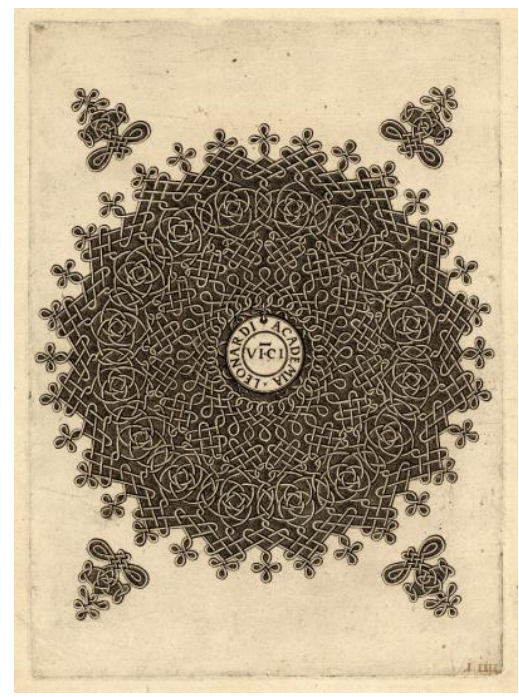

Figure 13. Étude d'entrelacs (L. de Vinci)

En effet, il se révèle peu à peu un maître exceptionnel dans ce domaine. En 1498, représentant $L a$ Dame à l'hermine, il a orné le haut de sa robe d'une bande de motifs d'entrelacs (figure 12), et plus tard, il orne aussi le haut de la robe de la Joconde. Plusieurs études d'entrelacs, réalisées entre 1495 et 1500, nous sont parvenues : six d'entre elles ont été gravées comme celle de la figure 13. Elles semblent être le résultat d'un tracé continu. Elles sont tout à fait remarquables.

Le filigrane parcourant les bandes de l'étole (figure 14), ne suit pas des lignes courbes comme celles de la figure 13, mais des segments de droites, parfois alignés, parfois inclinés, qui se chevauchent, remplissant l'espace des deux bandes, en constituant des formes géométriques facilement perceptibles: des rectangles, des hexagones allongés et des carrés sur la pointe. Ces formes qui se recouvrent partiellement, ne sont pas créées de façon indépendante, mais résultent de la conjonction des passages successifs du même filigrane. La répétition de ces motifs géométriques engendre des modules caractéristiques.

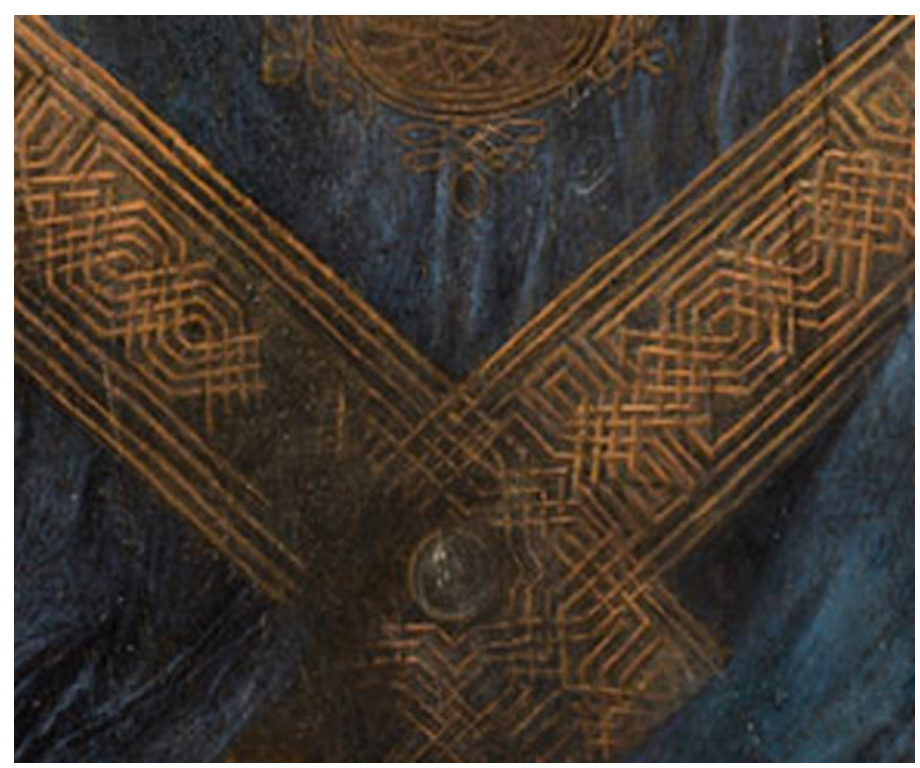

Figure 14. Les deux bandes de l'étole et les motifs formés par le filigrane, modélisées figure 15 


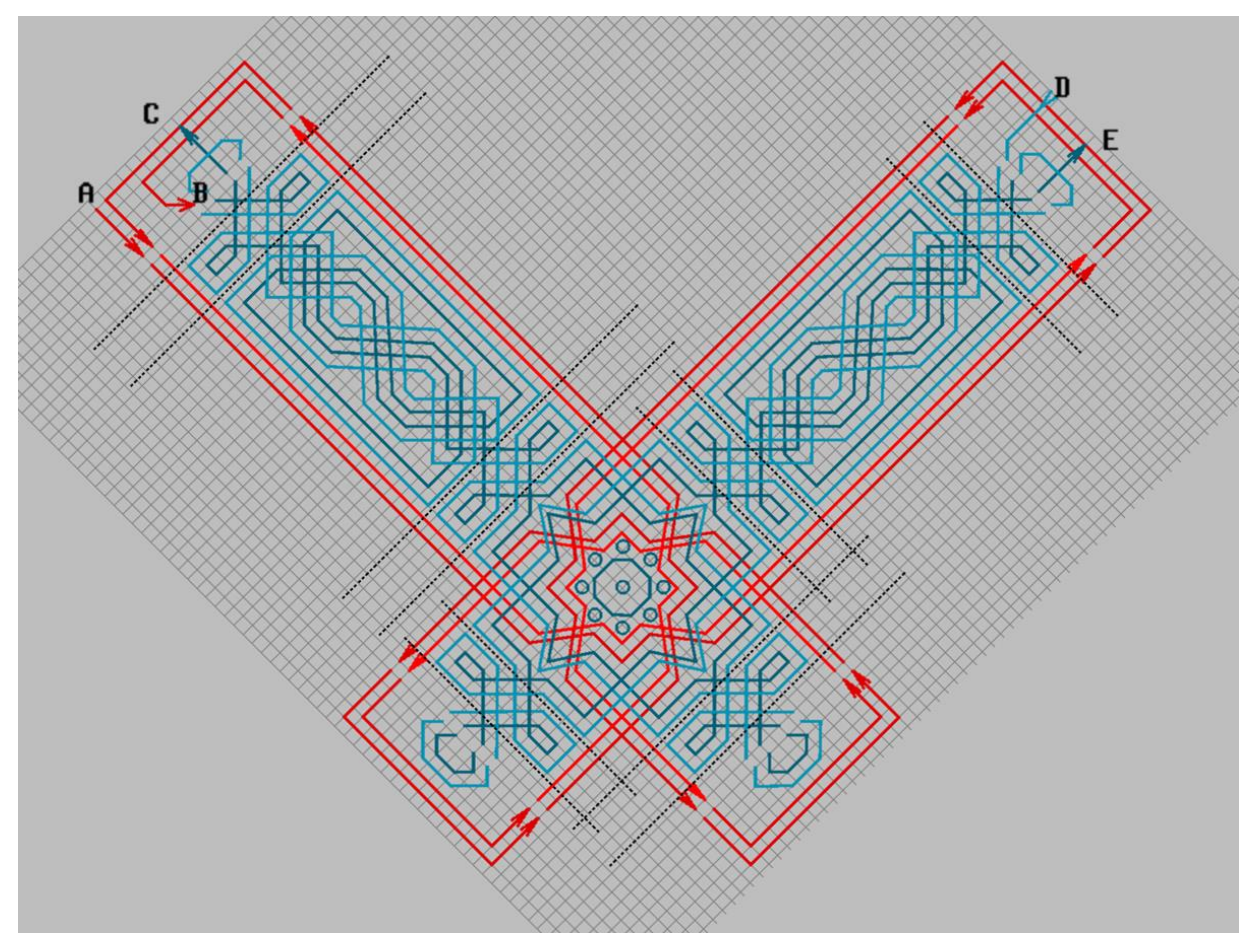

Figure 15. Représentation du cheminement du filigrane sur les bandes croisées de l'étole, et les différents modules, séparés par des lignes pointillées.

À l'intérieur de certains modules, la largeur de la bande est traversée par 16 passages du filigrane, qui sont régulièrement espacés, ce qui nous suggère la démarche suivie par Léonard.

Pour concevoir le chemin emprunté par le filigrane et sa complexité, Léonard a probablement pris comme support géométrique, un maillage carré (carré à cause du croisement). Ce maillage lui a permis d'établir la régularité des espacements et la périodicité des motifs. Nous avons tenté de reconstituer (figure 15) le chemin parcouru par le filigrane pour orner les deux bandes croisées de l'étole. Pour appréhender la complexité de sa démarche, nous avons décomposé artificiellement, le cheminement du filigrane en 4 tronçons de couleurs différenteds : rouge foncé, rouge, bleu clair et bleu foncé. Les deux filigranes de couleur rouge foncée délimitent la largeur des bandes.

De plus, nous avons distingué 5 modules différents (figure 16), qui ont mêmes entrée-sorties.

- le module croisement qui se comporte comme un «rond-point» de forme carrée pour les filigranes rouges, et un espace de transition pour les filigranes bleus.

- le module principal qui possède une symétrie gauche-droite et une symétrie haut-bas. Les deux filigranes rouges traversent directement le module à l'aller et au retour, et forment 4 passages. Les deux filigranes bleus suivent chacun, une boucle interne à l'aller, et au retour, de telle sorte qu'ils forment $2 \times 2 \times 3=12$ passages. Le filigrane fait donc 16 passages dans ce module.

- le module de transition possède aussi une double symétrie. Les entrées-sorties sont à la même hauteur mais leur pente est inversée.

- les deux modules terminaux qui assurent les entrées et les sorties, et permettent la continuité du trajet du filigrane. 

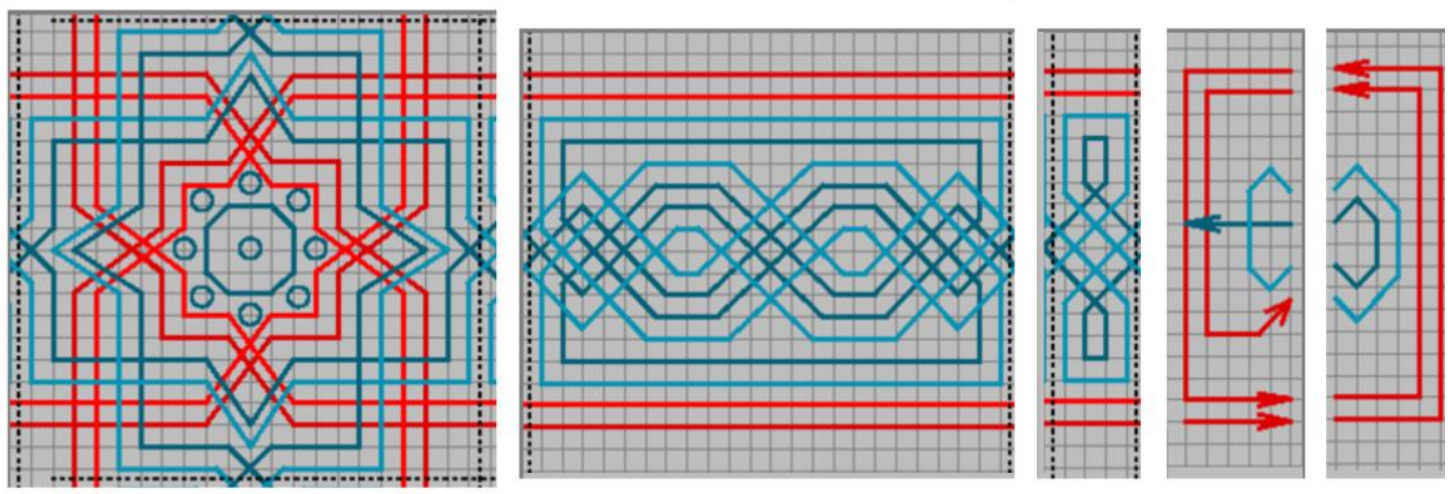

Figure 16. Les différents modules du trajet du filigrane. Les 3 premiers sont commutatifs.

Le trajet du filigrane est le suivant (figure 15). Le filigrane, d'abord codé en rouge foncé, fait le parcours d'une feuille quadrifoliée. Il part du point $\mathbf{A}$, au Nord-Ouest, tourne à gauche au rond-point, fait demi-tour au Nord-Est, puis tourne à gauche au rond point, fait demi-tour au Sud-Est, tourne à gauche au rond-point, fait demi-tour au Sud-Ouest, tourne à gauche au rond-point et se dirige vers le Nord-Ouest. Ainsi, après avoir suivi le bord des deux bandes, il devient rouge. Il effectue alors un trajet parallèle au premier, et à l'arrivée, en $\mathbf{B}$, il se transforme en un filigrane bleu clair.

Les filigranes bleus ont un parcours un peu plus complexe. Ils décorent l'intérieur des deux bandes (module 2). D'abord la bande Nord-Ouest Sud-est, puis la bande Nord-Est Sud-Ouest. Le filigrane bleu clair part du point B, situé au Nord-Ouest, traverse le module de transition, change de direction, ondule au centre du module principal, puis revient en arrière par le haut de ce module, et se dirige vers le module croisement qu'il traverse pour faire demi-tour au Sud-Est, repasser le module croisement et revenir vers le Nord-Ouest, où il se transforme en filigrane bleu foncé. Le filigrane bleu foncé effectue symétriquement un parcours semblable et sort en $\mathbf{C}$ au Nord-Ouest.

Les deux filigranes bleus parcourent ensuite la deuxième bande en partant du point $\mathbf{D}$ au Nord-Est.

Ainsi, malgré son apparente complexité, le trajet du filigrane est continu (un seul lever de plume!), et est par suite compatible avec le principe du tracé des entrelacs.

Cependant, Léonard n'a pas transcrit directement sur le patron, le trajet du filigrane de la figure 15 , car les bandes ne sont pas inclinées à $45^{\circ}$ (comme nous l'avons remarqué plus haut) mais à $43^{\circ}, 31$. Après avoir disposé le module croisement, il a reporté ensuite sur le patron, les modules décrits figure 16, de manière à constituer l'ensemble du trajet recherché. Le trajet du filigrane comme les contours sont percés de petits trous pour former le poncif. Le passage d'une ponce à travers ces trous reproduit les contours et le trajet du filigrane en pointillés sur la toile.

Évidemment, pour parvenir à un aspect plus naturel, l'artiste remplace les croisements en faisant passer le filigrane audessus de son passage précédent. Et pour atténuer la rigueur géométrique des modules construits sur le maillage, il adoucit les angles du tracé par une sorte de «sfumato» géométrique.

\section{Géométrie interne du Salvator Mundi (version de Ganay)}

Vers les années 1980 est apparue une autre version du Salvator Mundi, la version de Ganay.

Le Salvator Mundi dans la version de Ganay aurait été commandé par la duchesse Anne de Bretagne, épouse du roi de France Louis XII qui le donne au couvent des Clarisses de Nantes. Saisi à la Révolution, le tableau, identifié comme de Léonard de Vinci, il entre dans la famille du baron de 
Lareinty, président du Conseil général de la Loire-Inférieure. Il y reste jusqu'à sa vente par ses héritiers à une collectionneuse parisienne en 1902 qui le vend au marquis de Ganay en 1909.

Cette version ${ }^{28}$ peinte aussi sur une planche de noyer, est légèrement plus large. La toge est de couleur différente. Le Christ paraît moins à l'étroit. Son épaule gauche et sa manche droite sont mieux représentées.
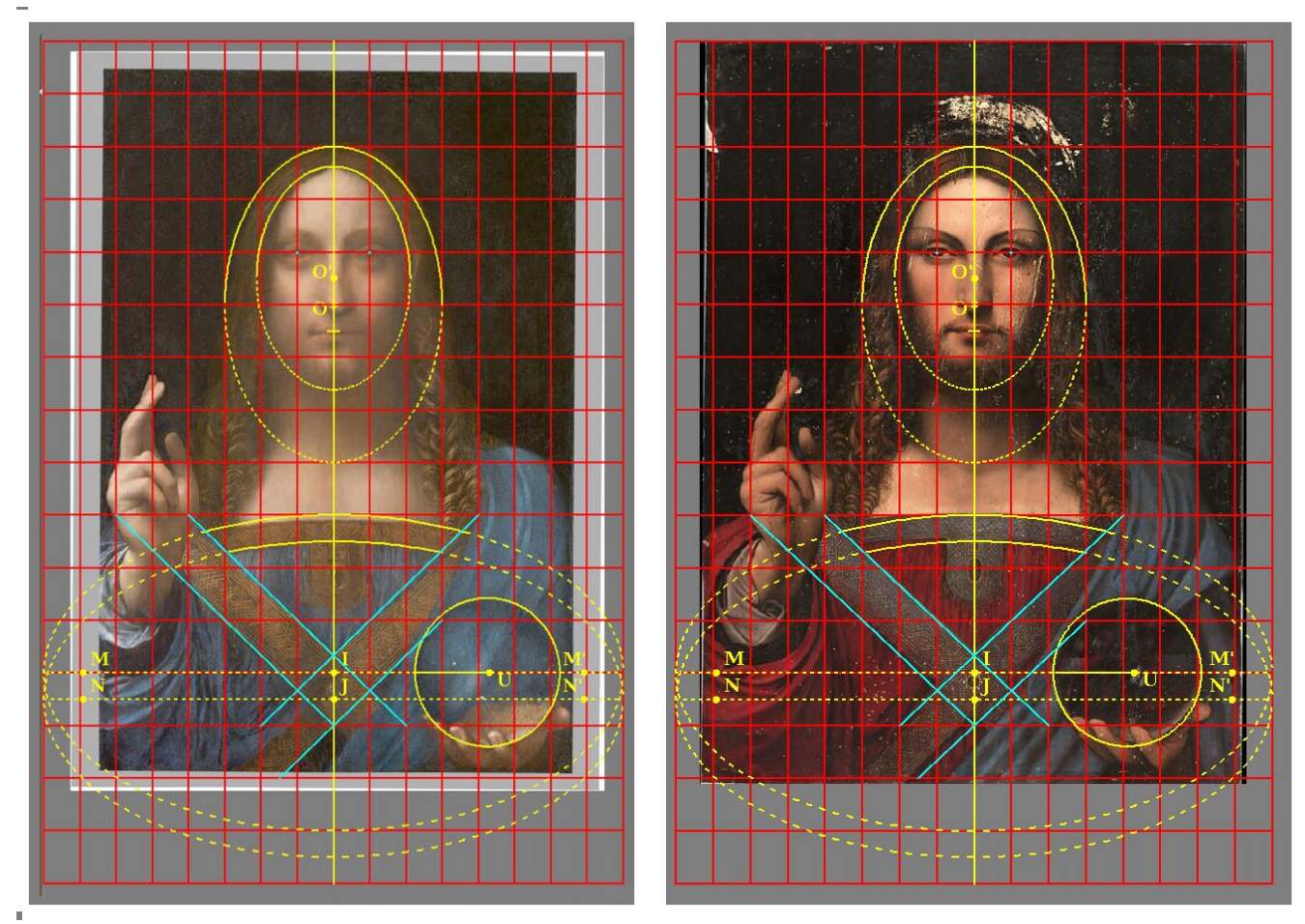

Figure 17. Géométrie interne du Salvator Mundi version Cook et celle de la version de Ganay

Nous avons appliqué à la version de Ganay, le même algorithme que celui qui nous a permis d'analyser la version Cook. Les deux versions présentent figure 17, la même géométrie interne.

Les deux versions ont donc été tracées à partir du même poncif, mais en effectuant dans les deux cas un déplacement vers l'axe vertical médian, de la partie du poncif correspondant à la sphère de cristal. Les éléments picturaux sont situés au même emplacement, et avec la même orientation.

Néanmoins, nous pouvons remarquer une petite différence : la sphère de cristal centrée au point $\mathbf{U}$, à une distance $\mathbf{I U}=\mathbf{4 , 1 0} \mathbf{m} \boldsymbol{h}$, est encore un peu plus rapprochée de l'axe vertical médian.
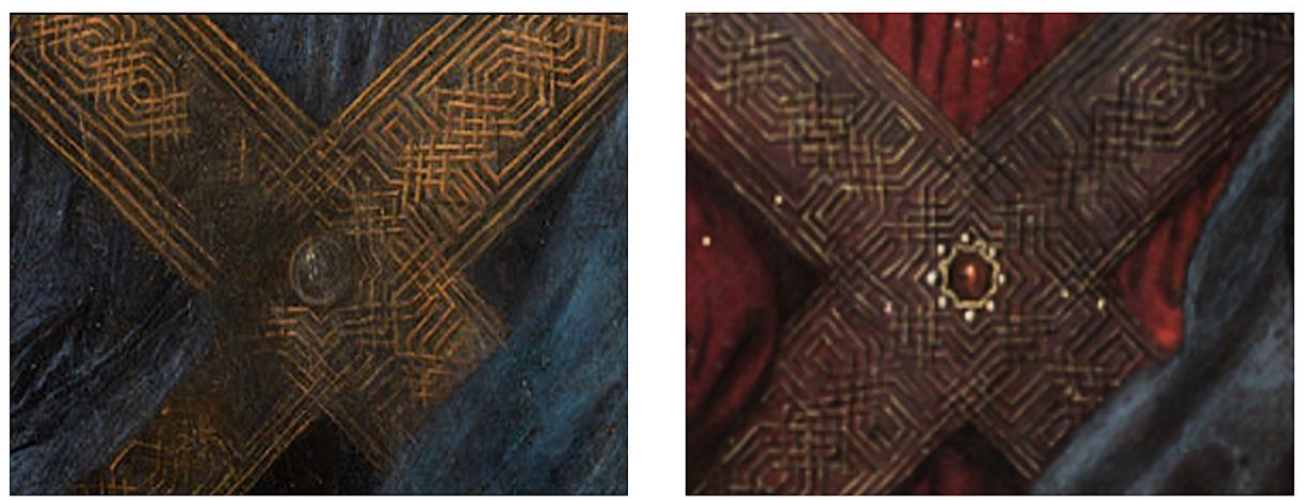

Figure 18. Détails des entrelacs des bandes croisées du Salvator Mundi version Cook et ceux de la version de Ganay

\footnotetext{
${ }^{28}$ https://fr.wikipedia.org/wiki/Fichier:School_of_Leonardo_da_Vinci,_Salvator_Mundi,_Museo_Diocesano,_Napoli.jpg. 
Les bandes croisées de l'étole montrent figure 18, les mêmes entrelacs situés aux mêmes endroits. Elles confirment l'utilisation d'un même poncif.

Existait-t-il une autre version tracée sur une planche plus large avec le même poncif, mais utilisé dans toute son intégralité ?

\section{Conclusion}

Le Salvator Mundi n'est pas un portrait. C'est la représentation du Christ, celle d'un homme qui a vécu parmi les hommes. Néanmoins, Léonard a suivi la même démarche que celle qu'il avait adoptée pour dresser la géométrie interne de ses cinq portraits féminins. Ainsi, Léonard, qui aimait donner au contour supérieur de la tête de chacun de ses personnages une forme elliptique, ne pouvait choisir qu'une forme elliptique particulière, idéale, unique pour modéliser la tête et le visage du Christ : celle d'une ellipse de type 1 , c'est-à-dire d'excentricité $\boldsymbol{\varepsilon}=\mathbf{1} / \sqrt{ } \mathbf{2}$. C'est grâce à la détection de cet arc d'ellipse, porteur de renseignements topographiques, que nous avons pu remonter facilement jusqu'au maillage et à la géométrie interne du Salvator Mundi. Le maillage harmonique 16x16 que nous avons détecté a sensiblement la même taille que celui qui a servi de support à la construction géométrique de la Joconde. Bien plus, ces deux compositions possèdent une particularité commune. Dans chacune d'elles, Léonard a introduit deux formes elliptiques semblables dont l'une a une surface deux fois plus petite que l'autre. Ces deux formes présentent ainsi dans chaque composition, une même consonance visuelle. Elles résonnent à l'octave. Enfin, pour illustrer le principe très léonardesque de la théorie dynamique de la géométrie, nous pouvons facilement concevoir, que c'est le mouvement de la main de l'artiste déroulant le filigrane sur les bandes croisées de l'étole, (en suivant le tracé de la figure 15), qui engendre petit à petit, au cours des allers et retours : les rectangles, les carrés sur la pointe et les hexagones allongés...

Ces processus géométriques sont propres au père de la Joconde. Ils nous incitent à considérer que le poncif (issu de la géométrie interne) qui a servi pour les deux versions du Salvator Mundi, est bien de la main de Léonard de Vinci.

\section{Bibliographie}

[1] Arasse D., Léonard de Vinci, Edition Hazan, Paris, 1993.

[2] Crettez J-P., Les supports de la géométrie interne des peintres : de Cimabue à G. de La Tour. Editions ISTE (2017).

[3] Crettez J-P., Openscience -Géométrie interne d'une "Nuit" de G. de La Tour : "L 'Apparition de l'ange à saint Joseph" (musée des beaux-arts, Nantes)

[4] Kemp M., Leonardo, Oxford, University Press, 2005. 